\title{
Are there monojets in high-energy proton-nucleus collisions?
}

\author{
N Borghini $\dagger$ and F Gelist \\ † Fakultät für Physik, Universität Bielefeld, Postfach 100131, D-33501 Bielefeld, \\ Germany \\ $\ddagger$ Service de Physique Théorique, CEA-Saclay, F-91191 Gif-sur-Yvette cedex, France
}

\begin{abstract}
We study high-energy proton-nucleus collisions within the Colour Glass Condensate framework, and compute the probabilities of having a definite number of scatterings in the nucleus with a momentum transfer larger than a given cut. Various properties of the distribution in the number of multiple scatterings are investigated, and we conclude that events with monojets are very unlikely, except for extreme values of the saturation scale $Q_{s}$.
\end{abstract}

\section{Introduction}

High-energy collisions of hadrons probe partons with very small $x$, whose occupation number becomes large, giving rise to the phenomenon of parton saturation [1]. The saturation is enhanced in a large nucleus, in which the nucleon wave functions overlap, and is expected to be revealed in high-energy collisions involving such a heavy nucleus.

Different frameworks implementing parton saturation have been developed, especially the McLerran-Venugopalan (MV) model [2,3] and later the so-called "Colour Glass Condensate" (CGC) [4]. A general feature in these approaches is the description of small- $x$ partons as classical Yang-Mills fields, radiated by the static large- $x$ colour sources. Computations of particle production in the collision of two saturated nuclei are then rather involved [5] and require numerical approaches. However, in interactions involving one small projectile, the colour sources describing the latter can be treated as weak: particle production can then be calculated by considering the relevant amplitude only at lowest order in this source, which allows one to obtain analytical expressions [6].

Hereafter, we shall focus on single-gluon production [7]. While it is known that the CGC framework automatically includes all multiple scatterings that lead to the production of a gluon with a high transverse momentum $k_{\perp}$, we shall study in further detail the distribution in the number of these scatterings [8], and more specifically, the scatterings with a momentum transfer larger than an arbitrary threshold $k_{\perp}^{\min }$.

\section{Models for the proton-nucleus interaction}

The number of gluons produced per unit of transverse momentum and per unit of rapidity in the collision between a proton and a saturated nucleus [7] involves the 
Fourier transform $C\left(\mathbf{k}_{\perp}\right)$ of a correlator of Wilson lines [9]. When the distribution of colour sources inside the nucleus only has Gaussian correlations, as is the case in the two models we shall consider hereafter, this correlator can be written in a form that has an interpretation in terms of independent multiple scatterings à la Glauber. In particular, the contribution to the gluon yield of cases in which the outgoing gluon has undergone exactly $n$ scatterings can be isolated. One can thus show that the conditional probability that a gluon that comes out with a momentum $\mathbf{k}_{\perp}$ has undergone $n$ scatterings with momentum transfers larger than a threshold $k_{\perp}^{\mathrm{min}}$ (and an arbitrary number of softer scatterings) reads [8]

$$
\begin{aligned}
P_{n}\left(\mathbf{k}_{\perp} \mid k_{\perp}^{\min }\right)= & \frac{\mathrm{e}^{-\mu_{0}^{2} \sigma_{\text {tot }}}}{C\left(\mathbf{k}_{\perp}\right)} \sum_{p=0}^{+\infty} \rho^{p+n} \int_{0}^{L} \mathrm{~d} z_{1} \int_{z_{1}}^{L} \mathrm{~d} z_{2} \cdots \int_{z_{p+n-1}}^{L} \mathrm{~d} z_{n+p} \int_{\Lambda}^{k_{\perp}^{\min }} \frac{\mathrm{d}^{2} \mathbf{k}_{1 \perp}}{(2 \pi)^{2}} \cdots \frac{\mathrm{d}^{2} \mathbf{k}_{p \perp}}{(2 \pi)^{2}}(2 \pi)^{2} \\
& \times \int_{k_{\perp}^{\min }} \frac{\mathrm{d}^{2} \mathbf{k}_{p+1}}{(2 \pi)^{2}} \cdots \frac{\mathrm{d}^{2} \mathbf{k}_{p+n}}{(2 \pi)^{2}} \delta\left(\mathbf{k}_{1 \perp}+\cdots+\mathbf{k}_{p+n_{\perp}}-\mathbf{k}_{\perp}\right) \sigma\left(\mathbf{k}_{1 \perp}\right) \cdots \sigma\left(\mathbf{k}_{p+n_{\perp}}\right),
\end{aligned}
$$

where $\rho$ is the number density of scattering centres in the nucleus, $L$ is the longitudinal size of the nucleus, $\mu_{0}^{2} \equiv \rho L$ is the density of scattering centres per unit of transverse area, and $\sigma\left(\mathbf{k}_{\perp}\right)$ is the differential cross-section of a gluon with a scattering centre (the integral of which is $\left.\sigma_{\text {tot }}\right)$. We shall present results obtained with two versions of this cross-section: either that, proportional to $k_{\perp}^{-4}$, of the MV model - in which the colour sources have a local Gaussian distribution —, or the slightly more complicated crosssection, $\propto\left(Q_{s}^{2} / k_{\perp}^{2}\right) \ln \left[1+\left(Q_{s}^{2} / k_{\perp}^{2}\right)^{\gamma}\right]$ with $\gamma \approx 0.64$, of the non-local Gaussian effective theory ("asymptotic model") that describes the gluonic content of a nucleus evolved to very small $x$ values [10]. These two models also yield different relationships between the saturation scale $Q_{s}$ and the scattering-centre density $\mu_{0}^{2}$ in equation (1).

\section{Results}

Instead of computing directly the probability $P_{n}$, equation (1), for each successive integer $n$, we rather calculate the generating function

$$
F\left(z, \mathbf{k}_{\perp} \mid k_{\perp}^{\mathrm{min}}\right) \equiv \sum_{n=0}^{+\infty} P_{n}\left(k_{\perp} \mid k_{\perp}^{\mathrm{min}}\right) z^{n},
$$

from which it is easy to extract the individual probabilities. This function, which has a quite simple analytic expression (equation (8) in reference [8]), also gives easy access to the average number of scatterings above the threshold $k_{\perp}^{\mathrm{min}}$, defined as

$$
N\left(k_{\perp} \mid k_{\perp}^{\mathrm{min}}\right) \equiv \sum_{n=1}^{+\infty} n P_{n}\left(k_{\perp} \mid k_{\perp}^{\mathrm{min}}\right),
$$

which is obviously given by the value at $z=1$ of the first derivative of equation (2). The distribution of the probabilities $P_{n}\left(k_{\perp} \mid k_{\perp}^{\min }\right)$ and the average number of recoils $N\left(k_{\perp} \mid k_{\perp}^{\mathrm{min}}\right)$, computed within the MV model, are displayed in figure 1 . First, the width 

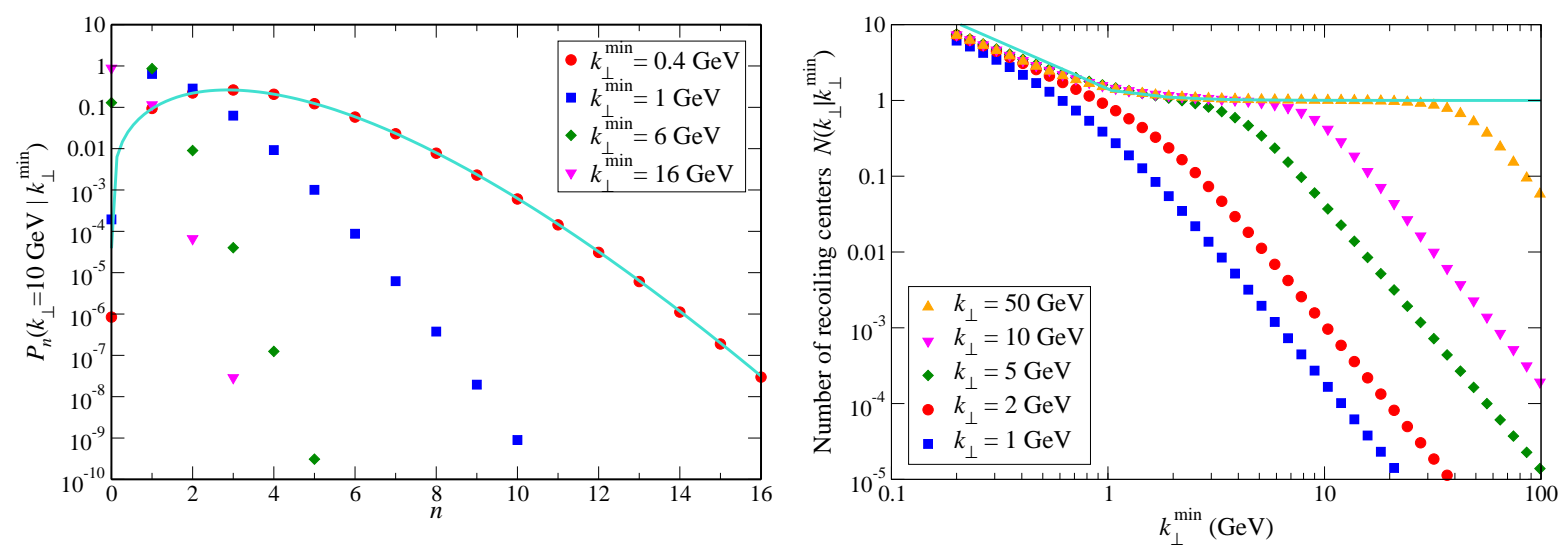

Figure 1. Left: distribution of the probabilities to have $n$ recoils when the produced particle acquires $k_{\perp}=10 \mathrm{GeV}$, for various values of the threshold $k_{\perp}^{\mathrm{min}}$. Right: number of recoils vs. the threshold $k_{\perp}^{\mathrm{min}}$, for various values of the acquired momentum $k_{\perp}$. Both panels assume the MV model with $Q_{s}^{2}=2 \mathrm{GeV}^{2}$.

of the multiplicity distribution decreases with increasing $k_{\perp}^{\mathrm{min}}$, which is quite natural, since it is less and less likely to have events in with a large number of recoils when $k_{\perp}^{\mathrm{min}}$ increases. One also sees that for all values of the threshold such that $Q_{s} \ll k_{\perp}^{\min } \lesssim k_{\perp}$, the most likely number of recoils is $n=1$, while $n=0$ is most likely when $k_{\perp}<k_{\perp}^{\min }$. This is paralleled by the fact that the number of recoils is very close to one for any value of $k_{\perp}^{\min }$ such that $Q_{s} \ll k_{\perp}^{\min } \lesssim k_{\perp}$ : when the gluon acquires a large momentum $k_{\perp}$, there is always one, and only one, hard recoil that provides most of this momentum. On the other hand, $N\left(k_{\perp} \mid k_{\perp}^{\mathrm{min}}\right)$ grows significantly at small $k_{\perp}^{\mathrm{min}}$, where it does not depend on $k_{\perp}$. This behaviour can be understood, since the generating function can be computed analytically when $k_{\perp}$ is much larger than the other two scales $Q_{s}$ and $k_{\perp}^{\min }$. One then finds that the probabilities $P_{n}$ follow a Poisson distribution shifted by one, corresponding to the compulsory hard scattering that provides most of the acquired momentum; the average value of this distribution is controlled by $Q_{s}$ and $k_{\perp}^{\min }$ only, independent of $k_{\perp}$. This analytic prediction is represented by the solid line in the figures.

The interpretation in terms of a single hard scattering that provides most of the momentum is further supported by the momentum distribution of the recoils, left panel of figure 2. Indeed, this distribution consists of a universal semi-hard component made of recoils with momenta $\lesssim Q_{s}$, and a second component peaked around $k_{\perp}^{\min }=k_{\perp}$. In other words, the most likely configuration is that of a di-jet, the high-momentum gluon with $k_{\perp}$ being balanced by a second hard parton with similar momentum, rather than a monojet, which would correspond to having many soft partons on the away side.

When the saturation scale $Q_{s}$ is varied (figure 2, right panel), the second, hard component around $k_{\perp}^{\min }=k_{\perp}$ is unchanged — as it should, since it is controlled by hard physics, not by saturation. On the other hand, the semi-hard region is affected by changes in $Q_{s}$, with more and more scatterings as $Q_{s}$ increases. This growth in the number of soft recoils, which is quite spectacular within the MV model (open symbols 

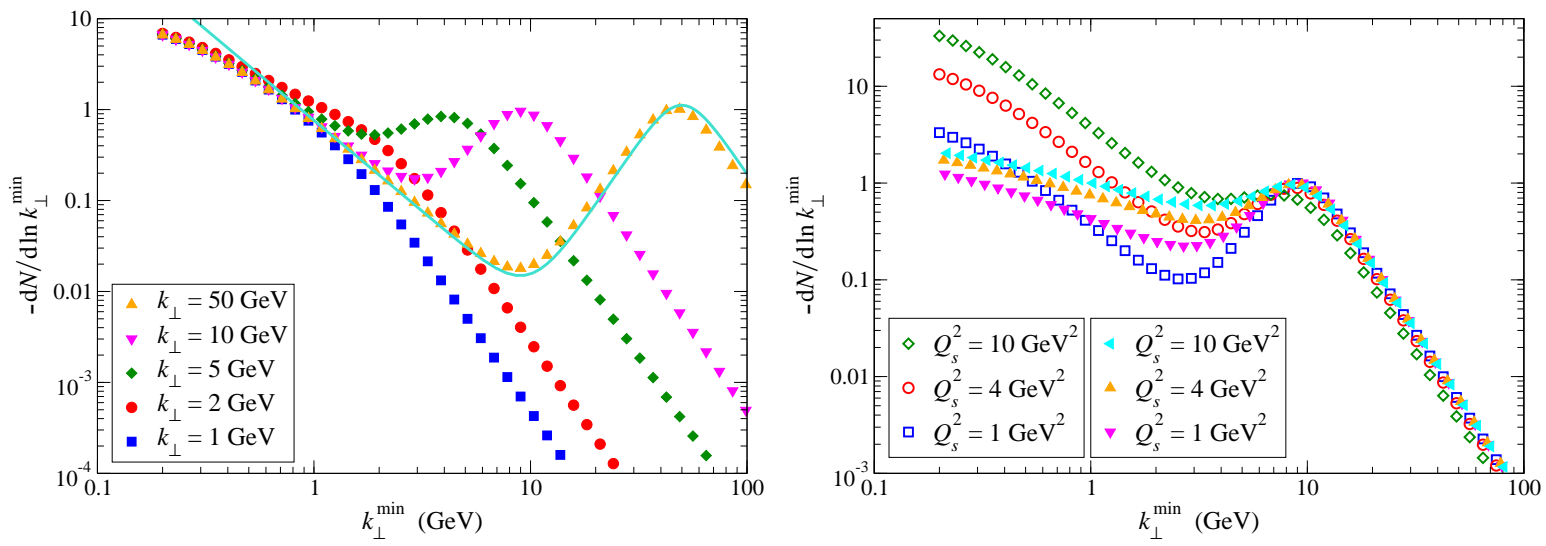

Figure 2. Distribution of the number of recoiling centres vs. the threshold $k_{\perp}^{\min }$. Left: for various values of the acquired momentum $k_{\perp}$, within the MV model with $Q_{s}^{2}=2 \mathrm{GeV}^{2}$. Right: dependence on the saturation $Q_{s}$ within the MV (open symbols) and asymptotic (full symbols) models, for a fixed value $k_{\perp}=10 \mathrm{GeV}$.

in figure 2), is however significantly reduced in the asymptotic model (full symbols). This lesser sensitivity is believed to reflect the presence of leading-twist shadowing in the latter model: first, the shadowing somehow "hides" the scattering centres from the passing gluon. In addition, since the effect of piling up more and more colour sources in the nucleus is tamed by the shadowing, the dependence on $Q_{s}$ is weaker in the asymptotic model than in the shadowing-free MV model.

Note also that the two-component structure of the momentum distribution of the recoils is less marked in the asymptotic model than in the MV model (figure 2, right panel). Especially, the dip between the two regions is filled up. This means that one should expect that the distribution of momenta in the "away-side jet" will become flatter as one probes the nucleus at smaller and smaller $x$ values. The di-jet structure, however, still persists, unless the value of the saturation momentum $Q_{s}$ becomes very large, opening the possibility of having a hard jet balanced by a large number of softer particles on the other side.

\section{References}

[1] Mueller A H and Qiu J 1986 Nucl. Phys. B 268427

[2] McLerran L D and Venugopalan R 1994 Phys. Rev. D 492233

[3] McLerran L D and Venugopalan R 1994 Phys. Rev. D 493352

[4] Iancu E and Venugopalan R 2004 Quark Gluon Plasma 3 ed R C Hwa and X N Wang (Singapore: World Scientific) p 249

[5] Gelis F 2007 (these proceedings)

[6] Jalilian-Marian J and Kovchegov Yu V 2006 Prog. Part. Nucl. Phys. 56104

[7] Blaizot J-P, Gelis F and Venugopalan R 2004 Nucl. Phys. A 74313

[8] Borghini N and Gelis F 2006 Phys. Rev. D 74054025

[9] Gelis F and Peshier A 2002 Nucl. Phys. A 697879

[10] Iancu E, Itakura K and McLerran L (2003) Nucl. Phys. A 724181 